\title{
Factors affecting systolic blood pressure trajectory in low and high activity conditions
}

\author{
Saiedeh Haji-Maghsoudi ${ }^{1}$, Azadeh Mozayani Monfared ${ }^{2}$, Majid Sadeghifar ${ }^{3}$, Ghodratollah Roshanaei ${ }^{1,4}$, \\ Hossein Mahjub $b^{1,5} *$ (D)
}

Received: 1 Jul 2020

Published: 26 Jul 2021

\section{Abstract}

Background: Typically, blood pressure dips during sleep and increases during daytime. The blood pressure trend is affected by the autonomic nervous system. The activity of this system is observable in the low and high activity conditions. The aim of this study was to assess the effect of individual characteristics on systolic blood pressure (SBP) across day-night under low and high activity conditions.

Methods: The samples were 34 outpatients who were candidates for evaluation of 24 hours of blood pressure with an ambulatory. They were admitted to the heart clinic of Farshchian hospital, located in Hamadan province in the west of Iran. The hourly SBP during 24 hours was considered as a response variable. To determine the factors effecting SBP in each condition, the hidden semi-Markov model (HSMM), with 2 hidden states of low and high activity, was fitted to the data.

Results: Males had lower SBP than females in both states. The effect of age was positive in the low activity state $(\beta=0.30 ; p<0.001)$ and negative in high activity state $(\beta=-0.21 ; \mathrm{p}=0.001)$. The positive effect of cigarette smoking on SBP was seen in low activity state $(\beta=5.02 ; p=0.029)$. The overweight and obese patients had higher SBP compared to others in high activity state $(\beta=11.60 ; p<0.001$ and $\beta=5.87 ; p=0.032$, respectively).

Conclusion: The SBP variability can be displayed by hidden states of low and high activity. Moreover, the effects of studied variables on SBP were different in low and high activity states.

Keywords: Activity, Body mass index, Ambulatory monitoring, Systolic blood pressure, Cigarette smoking

Conflicts of Interest: None declared

Funding: This study was supported by the grant No: 9710256427 from the Vice-Chancellor for Research and Technology of Hamadan University of Medical Sciences, Iran.

\section{*This work has been published under CC BY-NC-SA 1.0 license. \\ Copyright $\odot$ Iran University of Medical Sciences}

Cite this article as: Haji-Maghsoudi S, Mozayani Monfared A, Sadeghifar M, Roshanaei Gh, Mahjub H. Factors affecting systolic blood pressure trajectory in low and high activity conditions. Med J Islam Repub Iran. 2021 (26 Jul);35:95. https://doi.org/10.47176/mjiri.35.95

\section{Introduction}

Cardiovascular disease (CVD) and stroke are the leading causes of morbidity and mortality worldwide (1). About 17 million deaths occur due to CVD annually. Among heart diseases, at least $45 \%$ of deaths are related to hypertension each year (2). High blood pressure is an important public health concern that is also preventable $(3$, 4).

Blood pressure (BP) has fluctuations during a day-night.

Corresponding author: Dr Hossein Mahjub, mahjub@umsha.ac.ir

1. Department of Biostatistics, School of Public Health, Hamadan University of Medical Sciences, Hamadan, Iran

2. Department of Cardiology, School of Medicine, Farshchian Heart Center, Hamadan University of Medical Sciences, Hamadan, Iran

3. Department of Statistics, Faculty of Basic Sciences, Bu-Ali Sina University, Hamadan, Iran

4. Modeling of Noncommunicable Diseases Research Center, Hamadan University of Medical Sciences, Hamadan, Iran

5. Research Center for Health Sciences, Hamadan University of Medical Sciences, Hamadan, Iran
BP has the lower value at night, especially during sleeping. It starts to rise before waking up, then it has 2 peaks; the first: 2 or 3 hours after awakening; second: in early evening (5-7). This means the interindividual BP changes dynamically during 24 hours (8). These variations are influenced by the multilateral interaction of either external environmental and behavioral factors or the intrinsic mechanisms of cardiovascu lar regulation (9). The differ-

$\uparrow$ What is "already known" in this topic:

Various studies have examined the relationship between age, gender, body mass, and smoking on systolic blood pressure. However, in the studies, the constant time effect of the variables have been evaluated.

$\rightarrow$ What this article adds:

The time-varying effects of age, gender, body mass, and smoking on systolic blood pressure were investigated under low and high activity conditions during the day-night. 
ence in BP profiles in day to night is affected by both activity levels of individuals and cycles of sleep and wake during 24 hours. Some influential mechanisms for these variations include decreasing the activity of sympathetic system during night (10) and renal sodium (11). BP and its decline in sleep time are important risk factors for CVD (7). The prevalence of some cardiovascular events is greatest in early morning $(12,13)$. It is due to changes from sleep to the wakefulness state, which is associated with increased blood pressure, heart rate, and activity of the sympathetic nervous system (12).

One limitation of previous studies is considering fixed clock hours for awaking and sleep time, which leads to inaccurate definition of daytime and nighttime. Therefore, calculation of BP during the rest and activity states may not be realistic (7). In recent years, longitudinal studies have increased in widespread fields, especially in medical and epidemiological research areas (14). Unlike crosssectional studies in which the response is measured at a one-time point, in longitudinal studies, individual responses are collected at several time points (15). The main advantages of recording data at multiple time points are obtaining a more accurate evaluation of the outcome and effects of covariates during the time course. (16). In longitudinal studies, the temporal change of response is measured directly. In addition, some parts of changes are related to unobserved variables (17).

There are several approaches for analysis of longitudinal data, such as mixed models and marginal models (18). The time-varying unobservable variables can be covered by other approaches. One interesting approach in analyzing such data is using hidden Markov models (HMMs) (19). In recent years, HMMs have been welcomed by researchers in epidemiologic and medical studies. The HMMs consist of 2 observed and unobserved processes. The observed process is conditional on a hidden process with finite states. This means the observed value at any time is affected by a hidden state at the same time. In a special case, the state of hidden process at any time depends only on the state in the previous time (19).

In medical studies, hidden states can be a person's health status, which is not a well-defined quantity. Therefore, it seems reasonable that the health condition is not observed directly (20). The application of HMM in medical studies can be seen in analysis of hospital infection (21), electrocardiogram signal analysis (22), progression of liver cirrhosis (23), disease biomarker data (24), and risky teenage driving behavior (25). Hidden semi-Markov models (HSMMs) are generalization of HMMs. They are more flexible than HMMs in terms of occupancy distribution (26). The HSMMs have been used in many scientific areas such as pattern recognition $(27,28)$, rainfall $(29,30)$, and medical sciences $(31,32)$.

Usually, the diagnosis of hypertension and decisionmaking for treatment is based on daytime blood pressure (7). Compared with the traditional method, ambulatory BP monitoring measures the patients' systolic (SBP) and diastolic (DBP) pressure when they do regular daily activities without restriction during the day-night. This device usually records BP every half-hour during the day and hourly at night. The role of autonomic nervous system in the regulation of BP is important (33). The cardiovascular system is associated with the autonomic nervous system. The sympathetic and parasympathetic activities are determiners of high and low heart rates $(33,34)$. Therefore, activation of autonomic nervous system can be considered as a hidden process with low and high activity states for BP trend.

Based on our knowledge there is no comprehensive study to evaluate the effects of individual characteristics on BP trend in 24 hours for low and high activity periods. As HSMMs are able to evaluate the effects of covariates on response variable in different hidden states, the aim of this study was to evaluate factors affecting on 24-hour SBP variability under a dynamic model using HSMMs.

\section{Methods}

In this observational study, 34 outpatients who were candidate for ambulatory monitoring were included in the study. They were referred by a cardiologist to the clinic of Farshchian heart center hospital in Hamadan, a city in the west of Iran. They were candidates for assessment of their blood pressure using ambulatory monitoring in the first 9months of 2019. The patients were older than 18 years and provided their informed consent for participating in the study. The inpatients were excluded from the study. Their 24-hour blood pressures were recorded using an ambulatory monitoring on routine workdays. The study protocol was approved by the scientific committee of the Hamadan University of Medical Sciences. All the participants provided signed informed consent before the study.

The time-varying variables of SBP, DBP, and heart rate average were measured hourly based on the 24-hour ambulatory monitoring. The hourly SBP of each patient across 24 hours was considered as the response variable. The variables of age (year), gender, and body mass index (BMI), and history of cigarette smoking were the explanatory variables. The measured time was the official time.

The evaluation of biomarkers effects on BP in the alternation of the autonomic nervous system is of interest. Due to the longitudinal structure of the data, conventional analytical methods such a marginal models should be used (18). As mentioned earlier, the effect of hidden variables is not considered in conventional approaches. It is important to apply a model that accounts for fluctuations of BP during the day-night. We handled this issue by using HSMM.

\section{Statistical Analysis}

Two models were fitted to the data. In the first scenario, the marginal model using generalized estimating equations (GEE) was fitted to the dlata. Due to the nonlinearity of the trend changes in blood pressure during the daynight, the polynomial trajectories of time were included in the model. In the second scenario, to account for the effect of hidden states on SPB across day-night, HSMM was fitted to the data.

The model is defined as:

$$
\boldsymbol{y}_{i t}=\boldsymbol{X}_{i t}^{T} \boldsymbol{\beta}_{j}+\boldsymbol{\varepsilon}_{i t}
$$


where $\mathbf{y}_{i t}$ is the value of response variable for subject $i$ at time $\mathrm{t} ; \mathrm{i}=1, \ldots, \mathrm{n}$ and $\mathrm{t}=1, \ldots, \mathrm{T}$. $\mathbf{X}_{\mathrm{it}}$ is the covariate values at the same situation. $\boldsymbol{\beta}_{\mathrm{j}}$, is the $\mathrm{p} \times 1$ vector of regression coefficients under hidden state $\mathrm{j} ; \mathrm{j}=1, \ldots, \mathrm{J}$. Furthermore, $\boldsymbol{\varepsilon}_{\text {it }}$ indicates the error term for subject $\mathrm{i}$ at time $\mathrm{t}$.

Statistical analyses were performed using R freely software version 4.0.2. A program was written for the HSMM and the "geepack" package was used for applying the marginal model. The probability of type I error was considered .05 to evaluate the significance of variables.

\section{Results}

The sample included 34 outpatients, with a mean (standard deviation) age of 44.32 (15.67) years, ranging from 18 to 81 years. The majority of patients were female $(n=20)$. More than half of the patients were overweight $(n=18)$, of whom 12 were obese. Among the participants, 6 had the history of cigarette smoking (Table 1).

Figure 1 presents the SBP, DBP, and heart rate average across day-night. In the first scenario, marginal model using generalized estimating equations (GEE) was fitted to the data. By applying this approach, only the linear, quadratic, and cubic effects of time were significant. The positive effect of time showed an increasing trend of SBP $(p=0.004)$ during time. However, the negative significant effect of quadratic term of time showed a deceleration in an increasing trend $(p<0.001)$. The significant effect of cubic term of time $(p<0.001)$ revealed the reverse deceleration with increasing time (Table 2).

Table 3 represents the results of 2-state HSMM. In State 1 , which corresponds to high activity state, all variables except cigarette smoking history had significant effects on

\begin{tabular}{lcc} 
Table 1. Characteristics of the Outpatients' Participants & \\
\hline Variable & Level & Frequency (\%) \\
\hline Gender & Male & $14(41.18)$ \\
& Female & $20(58.82)$ \\
History of cigarette smoking & Yes & $6(17.65)$ \\
& No & $28(82.35)$ \\
BMI & Normal $\left(18.5-25 \mathrm{~kg} / \mathrm{m}^{2}\right)$ & $4(11.76)$ \\
& Overweight $\left(25-30 \mathrm{~kg} / \mathrm{m}^{2}\right)$ & $18(52.94)$ \\
& Obese $\left(>=30 \mathrm{~kg} / \mathrm{m}^{2}\right)$ & $12(35.29)$ \\
Heart rate & Mean & $\mathrm{SD}$ \\
Age (year) & 71.63 & 12.49 \\
\hline
\end{tabular}

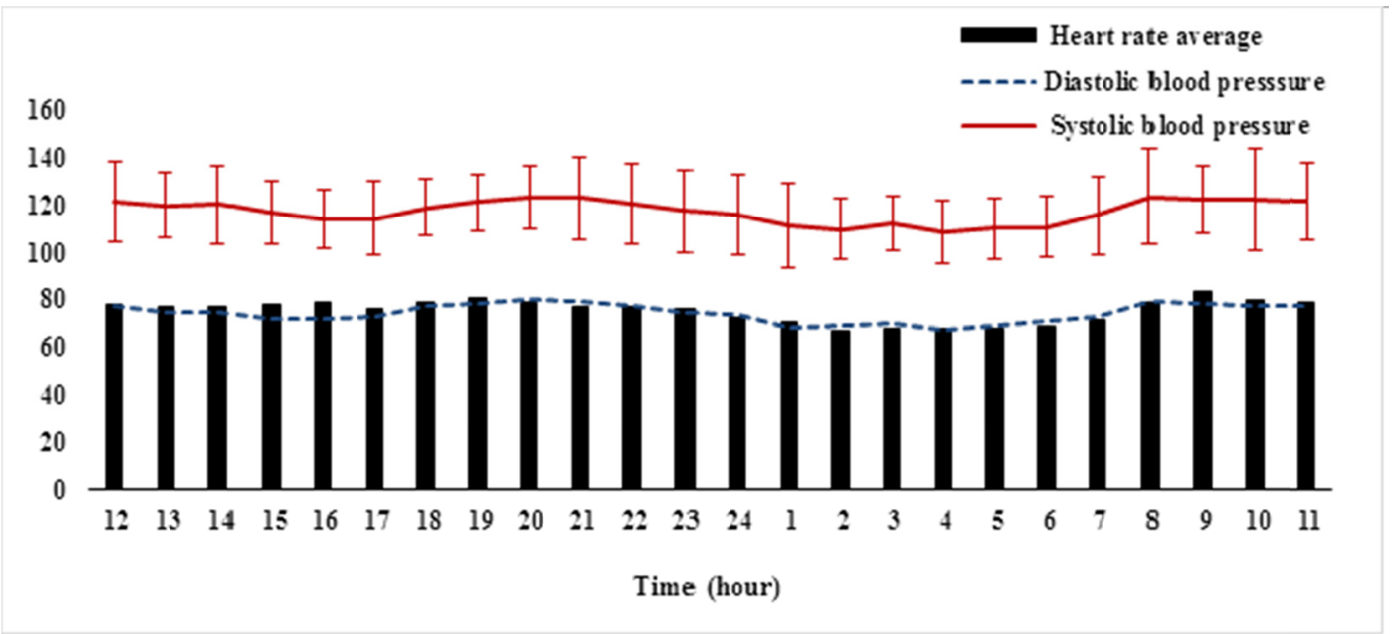

* Error bar indicates the standard deviation of systolic blood pressure

Fig. 1. Average of systolic and diastolic blood pressure and heart rate over day-night

Table 2. Effects of variables on systolic blood pressure (SBP) in marginal model

\begin{tabular}{lccc}
\hline Variable & Coefficient & SE & $\mathrm{p}$ \\
\hline Intercept & 115.61 & 4.70 & $<0.001$ \\
Time & 2.33 & 0.82 & 0.004 \\
Time`2 $^{\wedge}$ Time 3 & -0.30 & 0.08 & $<0.001$ \\
Age & 0.01 & 0.00 & $<0.001$ \\
Male gender & -0.07 & 0.09 & 0.394 \\
History of cigarette smoking & -4.87 & 4.01 & 0.224 \\
$\quad$ Normal (Ref) & 0.07 & 4.24 & 0.987 \\
BMI Overweight & - & & 0.150 \\
$\quad$ Obese & 6.42 & 4.45 & 0.514 \\
\hline
\end{tabular}




\begin{tabular}{|c|c|c|c|}
\hline Variable & Coefficient & $\mathrm{SE}$ & $\mathrm{p}_{1}$ \\
\hline \multicolumn{4}{|l|}{ High activity state } \\
\hline Intercept & 126.59 & 4.19 & $<0.001$ \\
\hline Age (year) & -0.21 & 0.07 & 0.0101 \\
\hline Male gender & -4.72 & 2.04 & 0.020 \\
\hline History of cigarette smoking & -0.30 & 2.35 & 0.897 \\
\hline Normal & - & & \\
\hline BMI Overweight & 11.60 & 2.98 & $<0.001$ \\
\hline Obese & 5.87 & 2.74 & 0.032 \\
\hline \multicolumn{4}{|l|}{ Low activity state } \\
\hline Intercept & 93.20 & 3.94 & $<0.001$ \\
\hline Age (year) & 0.30 & 0.07 & $<0.001$ \\
\hline Male gender & -6.21 & 1.85 & 0.0101 \\
\hline History of cigarette smoking & 5.02 & 2.30 & 0.029 \\
\hline Normal & - & & \\
\hline BMI Overweight & -0.12 & 2.89 & 0.967 \\
\hline Obese & 5.03 & 2.65 & 0.058 \\
\hline
\end{tabular}

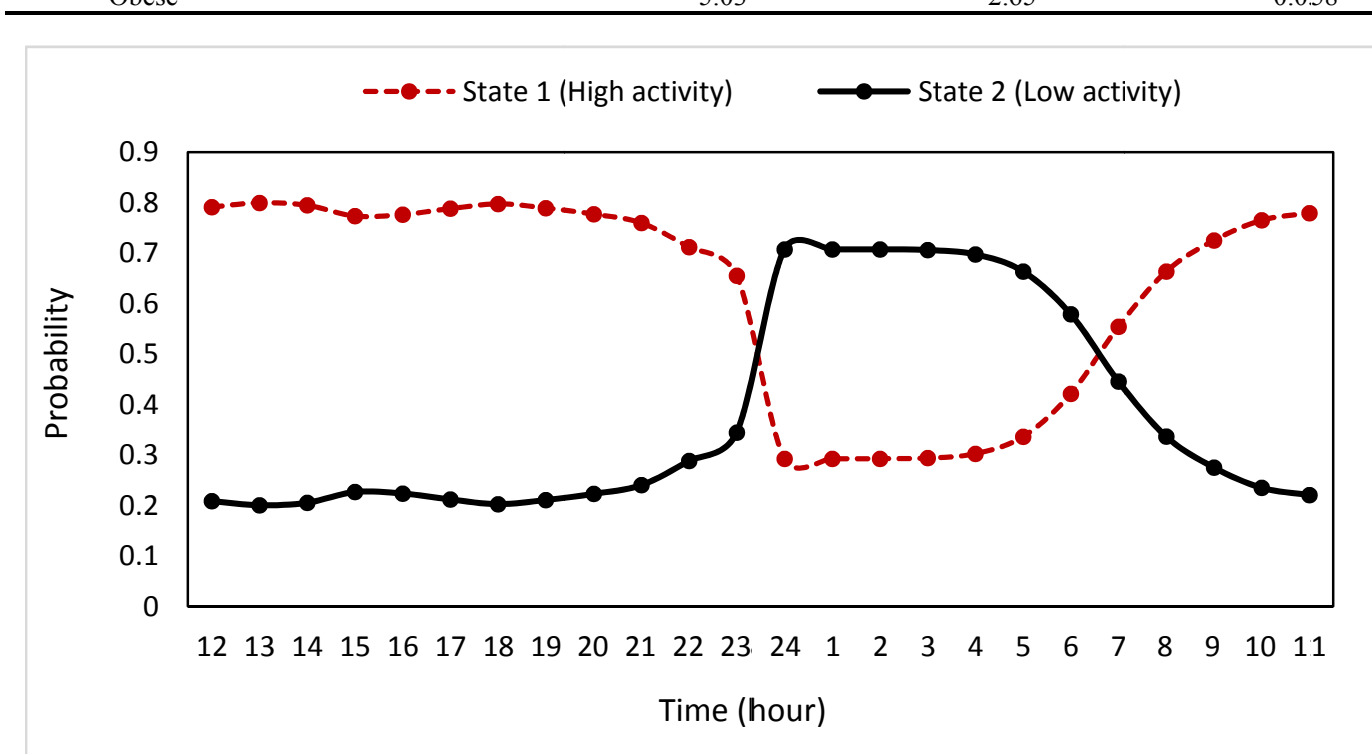

Fig. 2. The probability of being in each state during day-night

SBP. Age was negatively associated with SBP $(\mathrm{p}=0.001)$. Males had lower SBP mean than females $(p=0.020)$. The overweight and obese patients had more SBP than healthy participants $(\mathrm{p}<0.05)$. In State 2, which corresponds to low activity state, age had a positive effect on SBP $(p<0.010)$. Males had lower SBP average than females $(p=0.001)$. The history of cigarette smoking had positive significant effects on SBP $(p=0.029)$ (Table 3). Figure 2 presents the probability of belonging to each state during 24 hours. These probabilities were obtained using the HSMM. This figure is a good representative of low and high activity states.

\section{Discussion}

In the present study, 2 models were fitted to the data. The results of marginal model showed that the polynomial trajectories of time were significant. This means the trend of SBP is nonlinear during day-night. Moreover, HSMM, with 2 hidden states of low and high activity, was fitted to the data. The findings showed that males had lower SBP than females in both states. The effect of age was positive in low activity state and negative in the other state. Smokers had more SBP than nonsmokers in low activity state.
The increasing effect of BMI was seen only in high activity state. The effects of some risk factors, such as BMI and cigarettes smoking, on SBP were assessed in different studies (35-38). One of the causes of increased systolic blood pressure (SBP) in aging is due to increased arterial stiffness $(38,39)$. Comparison between the marginal and HSMM indicates that HSMM could reveal the significant effect of variables in time-varying conditions.

In a Korean study, the factors associated with the prevalence of hypertension were aging and high BMI. However, in different age groups, prevalence, and control of hypertension differed in men and women. They pointed out that the association between gender and prevalence and control of hypertension are unclear (40). A conducted study at the University of Jordan among healthy university students showed that SBP for males was higher than the females (41). In the study, participants were chosen from healthy young people, while in our study the average age of the participants was 44 years. They found that SBP is associated with BMI. Moreover, smokers had higher systolic blood pressure (41). These findings are in line with our results.

In recent studies using 24-hour outpatient blood pres- 
sure monitoring techniques, it has been found that blood pressure is higher in men than in women in similar ages (42). This is in contrast with our finding, which may be due to lack of age-matching for men and women in the present study. However, a closer look requires further study.

The mentioned studies focused on factors affecting hypertension. Based on the literature review, we could not find a study that evaluates the effect of variables in 2 different situations of high and low activity during day-night. As mentioned, the SBP is affected by some unobservable variables. This may be due to biological complexity of humans. Unobserved variables account for some of the variations within and between individuals. More tangible results can be obtained by considering these variables in the model. It is possible to obtain contradictory results in different studies when investigators perform crosssectional studies for evaluating the effects of risk factors on response. Because some individual characteristics such as heart rate and BP that change over time are affected by unmeasured factors, namely hidden states. For example, the association between smoking and BP in some studies is positive $(43,44)$ and it is inverse in the others $(45,46)$, which may be due to hidden states.

The strength of this study was the evaluation of the studied variables on SBP in low and high activity states using only one model. This can be important for clinical decisions because the applied model is able to explore low and high activity states for each person by considering his/her characteristics. Based on the results, the applied model has reasonably separated the 2 different situations of low and high activity states. One limitation of this study was that only the participants who referred to the heart clinic with symptoms of the disease were included in the study. Another limitation was the small sample size. Nonetheless, the small sample size was partially overcome by repeating the measurements in 24 hours. For better identification of the factors affecting blood pressure in low and high activity states, conducting a study for healthy population with a larger sample size can be valuable. Moreover, in this study, people from different age groups were included. Future studies can focus on specific age groups, such as adolescents, young adults, middleaged people, and the elderly. Furthermore, future studies can be conducted to evaluate the effects of important risk factors on variation of SBP.

\section{Conclusion}

Although some individual characteristics such as sex are fixed across time, their effects can be varying across time. The effects of some variables were different on SBP in low and high activity states. The present study revealed that the effects of cigarette smoking, age, and BMI are different in 2 states of low and high activity.

\section{Acknowledgement}

We gratefully thank the management and staff of the Farshchian heart center, especially Ms. Ranjbar and the staff of noninvasive diagnostic procedure unit who helped us to collect the data. Moreover, we would like to thank Ms. Dehdar for her assistant in data collection. This study was supported by the Vice Chancellor for Research and Technology of Hamadan University of Medical Sciences, Iran with (approved Ethical code: IR.UMSHA.REC. 1397.702.).

\section{Conflict of Interests}

The authors declare that they have no competing interests.

\section{References}

1. Hao G, Wang X, Treiber FA, Harshfield G, Kapuku G, Su S. Blood pressure trajectories from childhood to young adulthood associated with cardiovascular risk: results from the 23-year longitudinal Georgia stress and heart study. Hypertension. 2017;69(3):435-42.

2. Agostinis-Sobrinho C, Ruiz JR, Moreira C, Abreu S, Lopes L, Oliveira-Santos J, et al. Cardiorespiratory fitness and blood pressure: a longitudinal analysis. J Pediatr. 2018;192:130-5.

3. Yang Y, Dong B, Zou Z, Wang S, Dong Y, Wang Z, et al. Association between Vegetable Consumption and Blood Pressure, Stratified by BMI, among Chinese Adolescents Aged 13-17 Years: A National Cross-Sectional Study. Nutrients. 2018;10(4):451.

4. Turner JR, Viera AJ, Shimbo D. Ambulatory blood pressure monitoring in clinical practice: a review. Am J Med. 2015;128(1):1420 .

5. Verdecchia P, Angeli F, Mazzotta G, Garofoli M, Ramundo E, Gentile $\mathrm{G}$, et al. Day-night dip and early-morning surge in blood pressure in hypertension: prognostic implications. Hypertension. 2012;60(1):3442.

6. Hermida RC, Smolensky MH, Ayala DE, Portaluppi F, Crespo JJ, Fabbian F, et al. 2013 Ambulattory Blood Pressure Monitoring Recommendations for the Diagnosis of Adult Hypertension, Assessment of Cardiovascular and other Hypertension-associated Risk, and Attainment of Therapeutic Goals: Joint Recommendations from the International Society for Chronobiology (ISC), American Association of Medical Chronobiology and Chronotherapeutics (AAMCC), Spanish Society of Applied Chronobiology, Chronotherapy, and Vascular Risk (SECAC), Spanish Society of Atherosclerosis (SEA), and Romanian Society of Internal Medicine (RSIM). Chronobiol Int. 2013;30(3) :355-410.

7. Hermida RC, Ayala DE, Smolensky MH, Fernández JR, Mojón A, Portaluppi F. Sleep-time blood pressure: unique sensitive prognostic marker of vascular risk and therapeutic target for prevention. Sleep Med Rev. 2017;33:17-27.

8. Gosmanova EO, Mikkelsen MK, Molnar MZ, Lu JL, Yessayan LT, Kalantar-Zadeh $\mathrm{K}$, et al. Association of systolic blood pressure variability with mortality, coronary heart disease, stroke, and renal disease. J Am College Cardiol. 2016;68(13):1375-86.

9. Parati G, Ochoa JE, Lombardi C, Bilo G. Assessment and management of blood-pressure variability. Nature Rev Cardiol. 2013;10(3):143.

10. Narkiewicz K, Winnicki M, Schroeder K, Phillips BG, Kato M, Cwalina E, et al. Relationship between muscle sympathetic nerve activity and diurnal blood pressure profile. Hypertension. 2002;39(1):168-72.

11. Haynes WG. Role of leptin in obesity-related hypertension. Experimental physiology. 2005;90(5):683-8.

12. Bombelli M, Fodri D, Toso E, Macchiarulo M, Cairo M, Facchetti R, et al. Relationship among morning blood pressure surge, 24-hour blood pressure variability, and cardiovascular outcomes in a white population. Hypertension. 2014;64(5):943-50.

13. Butt M, Zakaria M, Hussain HM. Circadian pattern of onset of ischaemic and haemorrhagic strokes, and their relation to sleep/wake cycle. J Pakistan Med Assoc. 2009;59(3):129-32.

14. Fitzmaurice G, Davidian M, Verbeke G, Molenberghs G. Longitudinal data analysis: CRC Press; 2008.

15. Sutradhar BC, Rao RP. Inferemces in Longitudinal Count Data Models with Measurement Errors in Time Dependent Covariates. Sankhya B. 2016;78(1):39-65.

16. Keselman H, Algina J, Kowalchuk RK. The analysis of repeated 
measures designs: a review. Br J Math Stat Psychol. 2001;54(1):1-20.

17. STUDIES W. Overview of issues in the longitudinal analysis of respiratory data. Am J Respir Crit Care Med. 1996;154:S208-5211.

18. Gibbons RD, Hedeker D, DuToit S. Advances in analysis of longitudinal data. Ann Rev Clin Psychol. 2010;6:79-107.

19. Visser I. Seven things to remember about hidden Markov models: A tutorial on Markovian models for time series. J Math Psychol. 2011;55(6):403-15

20. Mathematical Psychology; New Findings from University of Amsterdam in the Area of Mathematical Psychology Published. Psychol Psychiatry J. 2012:125.

21. Cooper B, Lipsitch M. The analysis of hospital infection data using hidden Markov models. Biostatistics. 2004;5(2):223-37.

22. Andreao RV, Dorizzi B, Boudy J. ECG signal analysis through hidden Markov models. IEEE Transactions on Biomedical engineering. 2006;53(8):1541-9.

23. Bartolomeo N, Trerotoli P, Serio G. Progression of liver cirrhosis to HCC: an application of hidden Markov model. BMC Med Res Methodol. 2011;11(1):38.

24. Detilleux JC. The analysis of disease biomarker data using a mixed hidden Markov model (Open Access publication). Gene Select Evol. 2008;40(5):491.

25. Jackson JC, Albert PS, Zhang ZW. A two-state mixed hidden Markov model for risky teenage driving behavior. Ann Appl Stat. 2015;9(2):849-65.

26. $\mathrm{Yu}$ SZ. Hidden semi-Markov models. Artific Intellig. 2010;174(2):215-43.

27. Khelifa MO, Belkasmi M, Abdellah Y, ElHadj YO, editors. An accurate HSMM-based system for Arabic phonemes recognition. 2017 Ninth International Conference on Advanced Computational Intelligence (ICACI); 2017: IEEE

28. Ma Y-F, Jia X, Hu Q, Bai H, Guo C, Wang S. A New State Recognition and Prognosis Method Based on a Sparse Representation Feature and the Hidden Semi-Markov Model. IEEE Access. 2020;8:119405-20.

29. Sansom J, Thomson P. On rainfall seasonality using a hidden semiMarkov model. J Geophysic Res: Atmospheres. 2007;112(D15).

30. Sansom J, Thomson P. A hidden seasonal switching model for highresolution breakpoint rainfall data. Water Resources Res. 2010;46(8).

31. Kamson AP, Sharma L, Dandapat S. Multi-centroid diastolic duration distribution based HSMM for heart sound segmentation. Biomed Signal Process Control. 2019;48:265-72.

32. Oliveira J, Renna F, Mantadelis T, Coimbra M. Adaptive sojourn time HSMM for heart sound segmentation. IEEE $\mathrm{j}$ BIOMED HEALTH INFORM. 2018;23(2):642-9.

33. Saito I, Takata Y, Maruyama K, Eguchi E, Kato T, Shirahama R, et al. Association between heart rate variability and home blood pressure: The Toon Health Study. Am J Hyperten. 2018;31(10):1120-

34. Stein P, PK, Kleiger M, RE. Insights from the study of heart rate variability. Ann Rev Med. 1999;50(1):249-61.

35. D'Elia L, De Palma D, Rossi G, Strazzullo V, Russo O, Iacone R, et al. Not smoking is associated with lower risk of hypertension: results of the Olivetti Heart Study. Eur J Public Health. 2014;24(2):226-30.

36. Ghosh S, Mukhopadhyay S, Barik A. Sex differences in the risk profile of hypertension: a cross-sectional study. BMJ Open. 2016;6(7):e010085.

37. John U, Meyer C, Hanke M, Völzke H, Schumann A. Smoking status, obesity and hypertension in a general population sample: a cross-sectional study. J Assoc Physic. 2006;99(6):407-15.

38. Wu S, Jin C, Li S, Zheng X, Zhang X, Cui L, et al. Aging, arterial stiffness, and blood pressure association in Chinese adults. Hypertension. 2019;73(4):893-9.

39. Franklin SS. Systolic blood pressure: it's time to take control. Am J Hyperten. 2004;17(S3):49s-54s.

40. Choi HM, Kim HC, Kang DR. Sex differences in hypertension prevalence and control: analysis of the 2010-2014 Korea National Health and Nutrition Examination Survey. PLoS One. 2017;12(5):e0178334.

41. Alhawari HH, Al-Shelleh S, Alhawari HH, Al-Saudi A, Aljbour AlMajali D, Al-Faris L, et al. Blood pressure and its association with gender, body mass index, smoking, and family history among university students. Int J Hypertens. 2018;2018.

42. Reckelhoff JF. Gender differences in the regulation of blood pressure. Hypertension. 2001;37(5):1199-208
43. Bowman TS, Gaziano JM, Buring JE, Sesso HD. A prospective study of cigarette smoking and risk of incident hypertension in women. J Am College Cardiol. 2007;50(21):2085-92

44. Halperin RO, Michael Gaziano J, Sesso HD. Smoking and the risk of incident hypertension in middle-aged and older men. Am J Hypertens. 2008;21(2):148-52

45. Li G, Wang H, Wang K, Wang W, Dong F, Qian Y, et al. The association between smoking and blood pressure in men: a crosssectional study. BMC Public Health.. 2017;17(1):797.

46. Li H, Tong W, Wang A, Lin Z, Zhang Y. Effects of cigarette smoking on blood pressure stratified by BMI in Mongolian population, China. Blood Press. 201 0;19(2):92-7. 